\title{
Higher Levels of C-Reactive Protein and Ferritin in Patients with Overweight and Obesity and SARS-CoV-2-Related Pneumonia
}

\author{
Silvia Bettini ${ }^{a}{ }^{b}$ Giovanni Bucca ${ }^{b}$ Caterina Sensi ${ }^{b}$ Chiara Dal Prà ${ }^{b}$ \\ Roberto Fabris $^{b}$ Roberto Vettor ${ }^{a, b} \quad$ Luca Busetto $^{a, b}$ \\ ${ }^{a}$ Department of Medicine, University of Padova, Padova, Italy; ${ }^{\mathrm{b}}$ Center for the Study and the Integrated \\ Management of Obesity, Padova University Hospital, Padova, Italy
}

\section{Keywords}

Overweight - Obesity · Corona virus disease $19 \cdot$ Severe acute respiratory syndrome coronavirus $\cdot$ C-reactive protein

\begin{abstract}
Introduction: Overweight and obesity are associated with a more severe COronaVirus Disease 19 (COVID-19). Adipose tissue-related chronic inflammation could be a promoter for the occurrence of the cytokine storm that predicts aggravation of COVID-19. The primary aim was to investigate if this increased risk for more severe COVID-19 was associated with a higher inflammatory response. Methods: We enrolled patients $<75$ years old hospitalized in a medical COVID-19 ward with SARS-CoV-2-related pneumonia. Patients were classified according to BMI as normal weight, overweight, and obesity. Laboratory parameters were measured at admission and every second day during the hospital stay. Results: Ninety patients (64.4\% males; median age 61 years) were enrolled. Invasive mechanical ventilation (IMV) was needed in $9 \%$ of the patients with normal weight, in $32.4 \%$ of the patients with overweight, and in $12.9 \%$ of the patients with obesity ( $p=0.045)$. Maximal C-reactive protein (CRP) level during hospital stay was $92(48-122) \mathrm{mg} / \mathrm{L}$ in patients with
\end{abstract}

karger@karger.com www.karger.com/ofa

Karger $\stackrel{\text { ' }}{5}$
(C) 2021 The Author(s)

Published by S. Karger AG, Basel

This is an Open Access article licensed under the Creative Commons Attribution-NonCommercial-4.0 International License (CC BY-NC) (http://www.karger.com/Services/OpenAccessLicense), applicable to the online version of the article only. Usage and distribution for commercial purposes requires written permission. normal weight, $140(82-265) \mathrm{mg} / \mathrm{L}$ in patients with overweight, and 117 (67-160) $\mathrm{mg} / \mathrm{L}$ in patients with obesity $(p=$ 0.037). Maximal ferritin values were $564(403-1,379) \mu \mathrm{g} / \mathrm{L}$ in patients with a normal weight, $1,253(754-2,532) \mu \mathrm{g} / \mathrm{L}$ in patients with overweight, and $828(279-1,582) \mu \mathrm{g} / \mathrm{L}$ in patients with obesity $(p=0.015)$. Conclusion: Patients with overweight and obesity required more IMV and had higher peaks of CRP and ferritin than patients with normal weight during COVID-19.

(c) 2021 The Author(s) Published by S. Karger AG, Basel

\section{Introduction}

During the first year of the pandemic caused by the new severe acute respiratory syndrome coronavirus (SARS-CoV-2), overweight and obesity emerged among the factors associated with a more severe Corona Virus Disease 19 (COVID-19), being associated with an increased risk for hospitalization, invasive ventilation, intensive care unit (ICU) admission, and mortality [1]. Indeed, high BMI ranked after only advanced age as a risk factor for mortality [2], and its role as an indicator of disease severity was particularly strong in younger pa-
Correspondence to:

Luca Busetto, luca.busetto@ unipd.it 
tients $[3,4]$. Several mechanisms could explain this impact, including obesity-associated metabolic dysfunction, pulmonary problems, prothrombotic state, immune impairments, and comorbidities $[1,5]$. In particular, adipose tissue-related chronic inflammation has been postulated as a facilitator for the occurrence of the "cytokine storm" that predicts aggravation of COVID-19 [1, 5].

We previously observed that patients with overweight and obesity admitted in our medical ward for SARS-CoV2-related pneumonia required more frequently assisted ventilation than normal weight patients [6]. In this report, we investigated if this increased risk for more severe COVID-19 in patients with overweight and obesity aged $<75$ years was associated with a higher inflammatory response during the disease.

\section{Methods}

\section{Study Setting and Patients}

As previously reported [6], our Internal Medicine Department (Clinica Medica 3) was converted into a COVID-19 ward since the first wave of the SARS-CoV-2 epidemic in Italy. The ward admitted low-intensity care patients, in strict conjunction with the emergency department and the subintensive and intensive care units of our university hospital.

In this report, 185 patients hospitalized in our COVID-19 medical ward from March 23 to April 11, 2020, were studied, and 90 patients were included. Inclusion criteria were age $<75$ years, positivity to an oropharyngeal swab analyzed with real-time reverse transcriptase-polymerase chain reaction assays specific for SARSCoV-2, and confirmed diagnosis of COVID-19 pneumonia (by chest X-ray or CT, ultrasound scan, or autopsy confirmation). Data were extracted from the electronic medical record, and patients were assigned an alphanumeric identification code for privacy protection. Patients were classified according to their BMI as normal weight $\left(\mathrm{BMI}<25 \mathrm{~kg} / \mathrm{m}^{2}\right)$, overweight $\left(25 \mathrm{~kg} / \mathrm{m}^{2} \leq \mathrm{BMI}<30\right.$ $\left.\mathrm{kg} / \mathrm{m}^{2}\right)$, and obesity $\left(\mathrm{BMI} \geq 30 \mathrm{~kg} / \mathrm{m}^{2}\right)$. Data on sex, age, BMI, and baseline comorbidities (type 2 diabetes, hypertension, dyslipidemia, cardiovascular diseases, chronic respiratory disease, chronic kidney disease, chronic liver disease, autoimmune diseases, cancer, and dementia) were collected. Need for invasive mechanical ventilation (IMV), need for staying in ICU, and death were considered as indexes of COVID-19 severity and outcomes. The length of stay was calculated as the difference between the date of hospital admission and the date of hospital discharge or death. The time (in days) between the onset of COVID-19 symptoms and admission to the hospital was also recorded.

\section{Biochemical Measurements}

According to the clinical protocol used in our hospital for COVID-19 patients, the following laboratory parameters were measured at admission and every second day during the hospital stay: total white blood cells, lymphocytes, hemoglobin, platelets, creatinine, fasting plasma glucose and capillary plasma glucose during the stay, aspartate aminotransferase, alanine aminotransferase, gamma-glutamyl transferase, troponin I, brain natriuretic peptide, lactic dehydrogenase, D-dimer, IL-6, ferritin, and C-reactive protein (CRP). All tests were performed with standard diagnostic methods at the Padova University Hospital clinical laboratory. For the purpose of the present study, we collected the value at admission and the maximal or minimal value (depending on clinical significance) observed during the hospital stay for each variable. The time (in days) between the onset of COVID-19 symptoms and the day in which the maximal or minimal value was observed for each biochemical variable was also recorded.

\section{Statistical Analysis}

All variables were tested by the normal test (Shapiro-Wilk test) and equal variance test (Brown-Forsythe). One-way analysis of variance was used when normality test and equal variance test have been passed (data are presented as mean values \pm standard deviations) or, if not, the Kruskal-Wallis one-way analysis of variance on ranks (data are presented as median value [25th-75th percentile]). $\chi^{2}$ test (or Fisher's exact test) was carried out for categorical variables, and data were expressed as frequency (percentage). A $p$ value level $<0.05$ was considered significant. All statistical analyses were performed with the Systat Software SigmaPlot v.14 (Systat Software; Adalta, Arezzo, Italy).

\section{Results}

The baseline characteristics, clinical data, and outcomes of the 90 patients hospitalized for SARS-CoV2-related pneumonia in our COVID-19 medical ward are reported in total and according to BMI classes in Table 1. The patients were predominantly males $(64.4 \%)$ with a median age of 61 (interquartile range: $56-69$ ) years. Mean BMI was $28.4 \pm 4.7 \mathrm{~kg} / \mathrm{m}^{2}$ (interquartile range: $25.2-30.7$ $\mathrm{kg} / \mathrm{m}^{2}$ ). IMV was needed in 18 patients $(20.0 \%)$ and admission to the ICU in 22 patients (24.4\%). Five patients (5.5\%) died during hospitalization.

Among the 90 patients, $22(24.4 \%)$ had a normal weight, 37 (41.1\%) were affected by overweight, and 31 (34.4\%) by obesity. No significant differences in age and sex were observed between the 3 BMI classes. Hypertension was more prevalent in patients with overweight and obesity $(p=0.025)$, and respiratory chronic diseases were more prevalent in the obesity group $(p=0.011)$. No other significant differences in the rate of comorbidities were observed. IMV was needed in $9 \%$ of the patients with a normal weight, in $32.4 \%$ of the patients with overweight, and in $12.9 \%$ of the patients with obesity $(p=0.045)$. Admission to the ICU was requested in $9.0 \%$ of the patients with a normal weight, in $35.1 \%$ of the patients with overweight, and in $22.6 \%$ of the patients with obesity $(p=0.076)$. Death rate was not significantly different among the 3 groups (Table 1). No 
Table 1. Baseline characteristics, clinical data, and outcomes in 90 patients hospitalized for SARS-CoV-2-related pneumonia in a COVID-19 medical ward

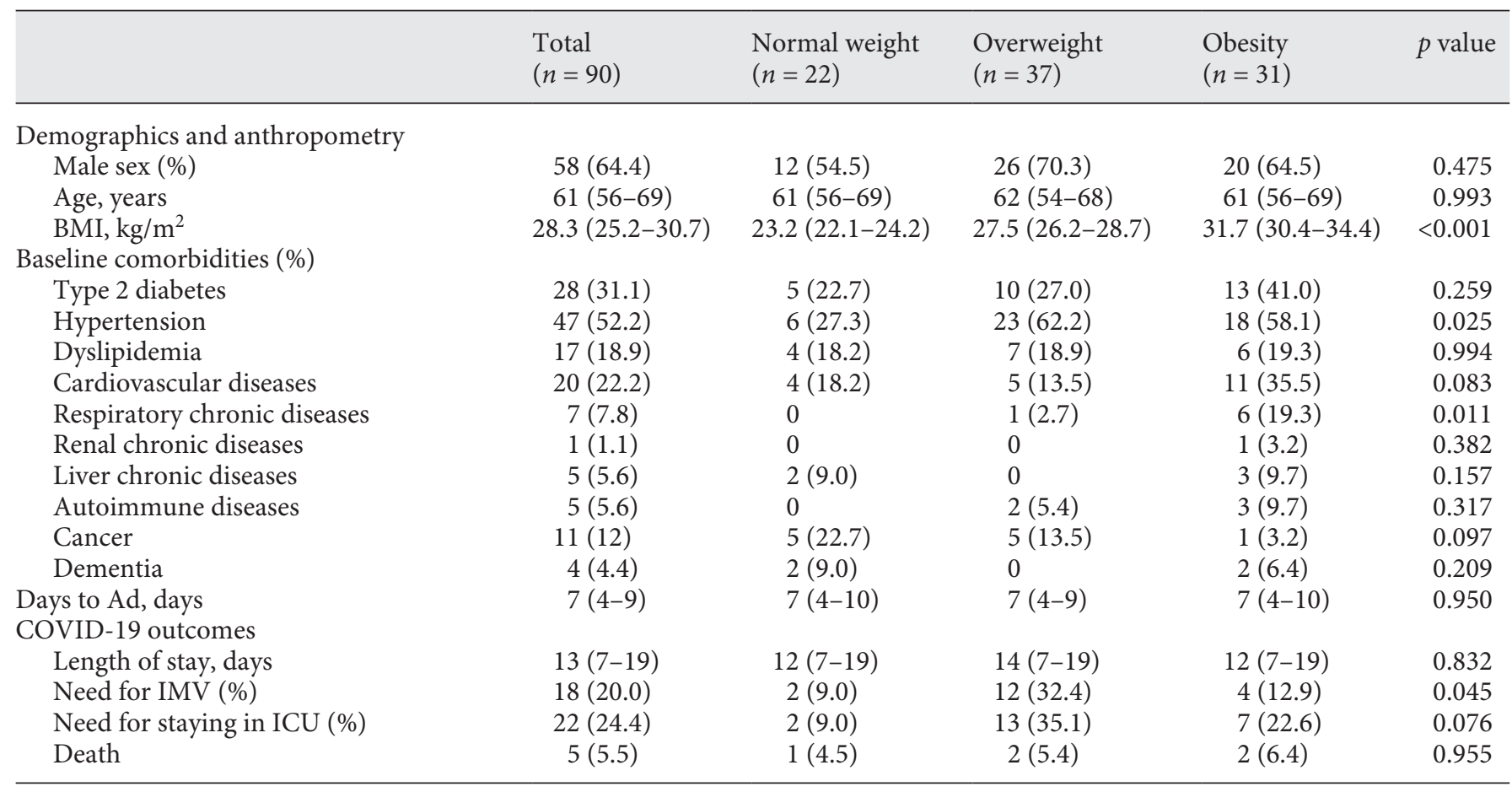

Data are presented as median (interquartile range) for continuous variables and as frequency (percentage) for categorical variables. Days to admission (Ad) were calculated as the difference between the date of onset of symptoms and the date of hospital admission. Length of stay was the difference between the date of hospital admission and the date of hospital discharge or death. BMI, body mass index; IMV, invasive mechanical ventilation; ICU, intensive care unit.

significant differences were observed between the 3 BMI classes in the time from the onset of symptoms to the hospital admission or in the length of hospital stay (Table 1).

The levels of routinely measured biochemical parameters in the 90 patients hospitalized for SARS-CoV-2-related pneumonia in our COVID-19 medical ward are reported according to BMI classes in Table 2. For any biochemical parameter, the level detected at admission and the maximal or minimal levels detected during the hospital stay (depending on clinical significance) are reported. We did not observe significant differences in CRP levels at admission in patients with normal weight, overweight, or obesity ( $p=0.219$ ) (Table 2; Fig. 1A). However, a significant difference became evident when we considered maximal levels of CRP during the hospitalization. Maximal CRP levels were $92(48-122) \mathrm{mg} / \mathrm{L}$ in patients with a normal weight, $140(82-265) \mathrm{mg} / \mathrm{L}$ in patients with overweight, and $117(67-160) \mathrm{mg} / \mathrm{L}$ in patients with obesity ( $p=0.037$ ) (Table 2; Fig. 1B). Ferritin values showed a statistically significant difference between the 3 BMI cat- egories, both considering levels at admission (443 [308$561] \mu \mathrm{g} / \mathrm{L}$ in patients with a normal weight, 892 [558$1,465] \mu \mathrm{g} / \mathrm{L}$ in patients with overweight, and 734 [178$1,008] \mu \mathrm{g} / \mathrm{L}$ in patients with obesity $[p=0.012$ ] [Table 2 ; Fig. 1C]) and maximal levels during hospitalization (564 $[403-1,379] \mu \mathrm{g} / \mathrm{L}$ in patients with a normal weight, 1,253 $[754-2,532] \mu \mathrm{g} / \mathrm{L}$ in patients with overweight, and 828 $[279-1,582] \mu \mathrm{g} / \mathrm{L}$ in patients with obesity $[p=0.015]$ [Table 2; Fig. 1D]). No significant differences between the 3 BMI groups were observed in the time (in days) between the onset of COVID-19 symptoms and the day in which the maximal value of CRP (9 [7-13] days in normal weight, 11 [8-14] days in overweight, and 10 [8-13] days in obesity) or ferritin (12 [9-14] days in normal weight, 11 [8-15] days in overweight, and 12 [9-17] days in obesity) was reached. No other significant differences were observed between BMI classes for all the other biochemical parameters, both at admission and as maximal or minimal values (Table 2), or in the times in which the maximal or minimal value of each parameter was reached (data not shown). 
Table 2. Biochemical parameters at admission and during the hospital stay in 90 patients hospitalized for SARS-CoV-2-related pneumonia in a COVID-19 medical ward

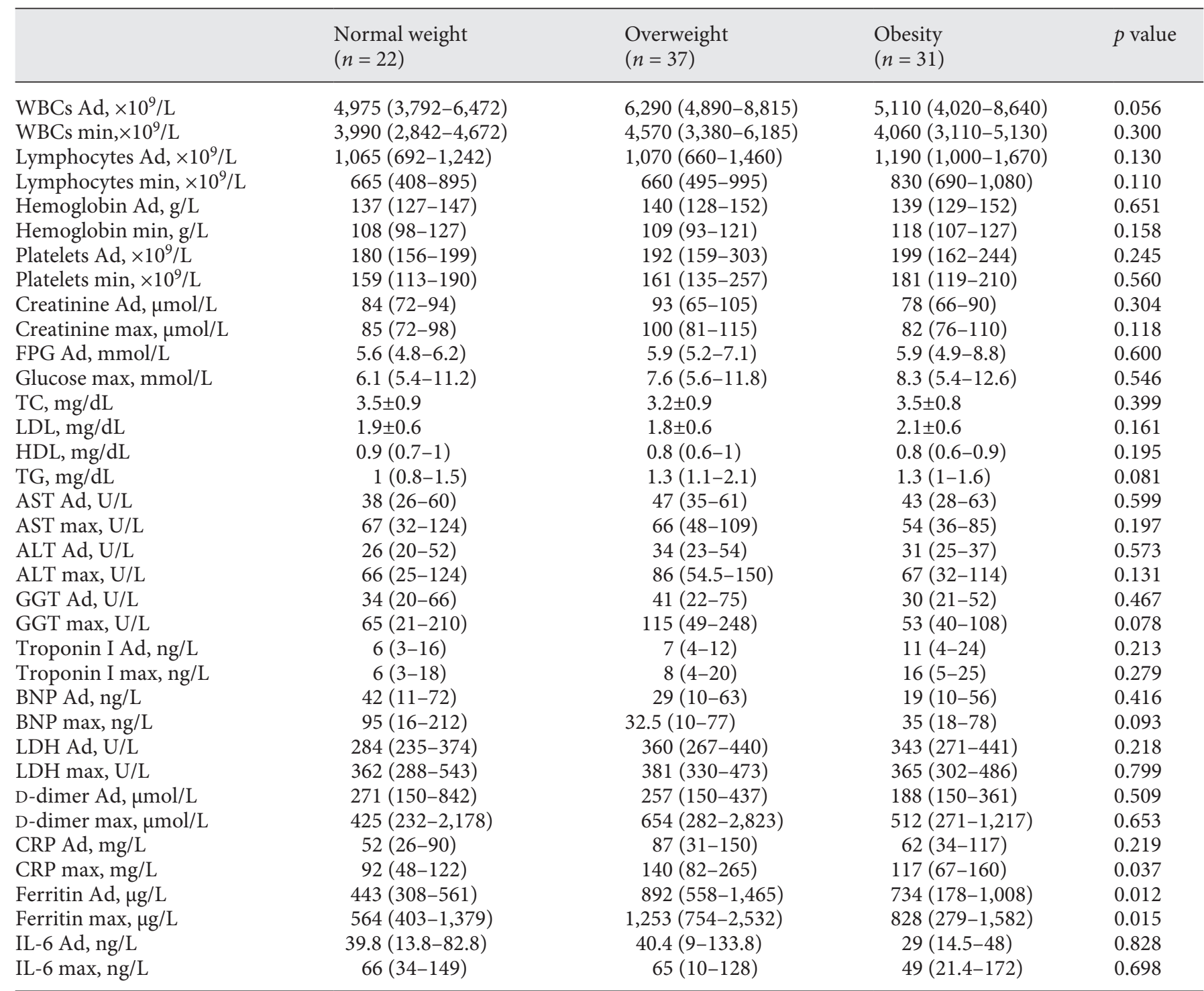

Data are presented as median (interquartile range). For any biochemical parameter, the level detected at admission and the maximal or minimal levels detected during the hospital stay ( $\mathrm{max} / \mathrm{min}$ ) are reported. WBCs, white blood cells; FPG, fasting plasma glucose; TC, total cholesterol; LDL, low-density lipoprotein-cholesterol; HDL, high-density lipoprotein-cholesterol; TG, triglycerides; AST, aspartate aminotransferase; ALT, alanine aminotransferase; GGT, gamma-glutamyl transferase; BNP, brain natriuretic peptide; LDH, lactic dehydrogenase; CRP, C-reactive protein; IL-6, interleukin-6.

\section{Discussion}

In this study, we confirmed our previous observations of a more severe COVID-19 in patients with overweight and obesity belonging to a cohort of Italian patients hospitalized in a dedicated medical ward with a diagnosis of SARS-CoV-2-related pneumonia [6]. In particular, we demonstrated that patients with over- weight and obesity aged $<75$ years displayed an increased severity of the viral disease, with a major need for IMV, as compared with the normal weight patients of the same age group. This increased severity of COVID-19 was accompanied by a greater activation of the inflammatory response, as demonstrated by higher $\mathrm{CRP}$ and ferritin values. 


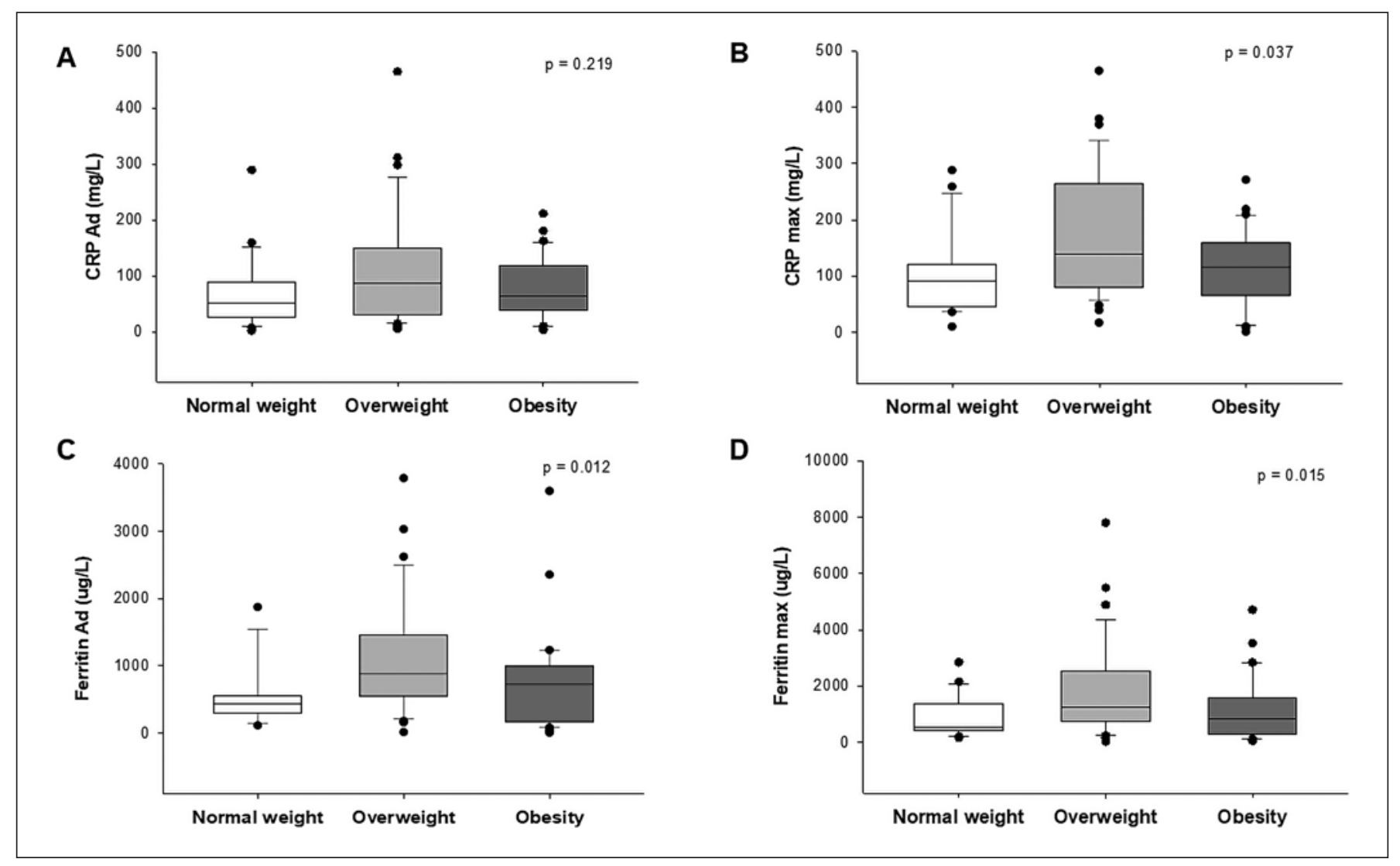

Fig. 1. Levels at Ad and max during hospitalization for CRP (A, B) and ferritin (C, D) in 90 patients hospitalized for SARS-CoV-2 related pneumonia. Patients were divided according to BMI into patients with a normal weight $\left(\mathrm{BMI}<25 \mathrm{~kg} / \mathrm{m}^{2}\right)$ (white bar), overweight $\left(25 \mathrm{~kg} / \mathrm{m}^{2} \leq \mathrm{BMI}<30 \mathrm{~kg} / \mathrm{m}^{2}\right)$ (gray bar), and obesity $\left(\mathrm{BMI} \geq 30 \mathrm{~kg} / \mathrm{m}^{2}\right)$ (black bar). $p$ value was determined by Kruskal-Wallis one-way analysis of variance on ranks. CRP, C-reactive protein; Ad, admission; max, maximal levels.

Since preliminary observations reporting a link between BMI and severity of COVID-19 at the very beginning of the epidemic in Europe [6, 7], several other larger studies confirmed the role of obesity among the factors associated with a more severe COVID-19, being associated with an increased risk for hospitalization, invasive ventilation, ICU admission, and mortality [1]. Obesity ranks second only to age as a risk factor for severe COVID-19 [2], and, thus, its role is particularly important in younger patients $[3,4]$. In this report in 90 hospitalized patients, with the exclusion of the older ones, we confirmed a risk of a more severe disease in patients with overweight and obesity. In fact, we showed an increased need for IMV and, even if not reaching a statistically significance, a greater need for staying in ICU in patients with overweight and obesity.

Several mechanisms could be involved in linking obesity with worse clinical outcome in patients with CO-
VID-19, including impaired ventilation, reduced cardiorespiratory fitness and obesity-associated metabolic complications, procoagulant state, dysfunctional immune response, and chronic state of low-grade inflammation [5]. In particular, severity of COVID-19 has been associated with the occurrence of the so-called "cytokines storm," and severe cases of SARS-CoV-2 disease had markedly higher levels of CRP and other inflammatory cytokines than cases of moderate severity [8]. Moreover, despite increased basal levels of proinflammatory mediators, obesity-associated inflammation is paradoxically associated with attenuated innate immune response to both bacterial and viral infections [9]. In our study, the higher peak levels of CPR and ferritin observed in patients with overweight and obesity could be an indirect confirmation of the importance of inflammatory pathways in explaining the link between adipose tissue inflammation and severity of COVID-19. IL-6 levels did not differ among the $3 \mathrm{BMI}$ subgroups, and in fact 
median values were moderately high in all patients. We may suggest that this inflammatory parameter, being a high sensible parameter to detect inflammation, was not accurate to identify a higher inflammatory response in patients belonging to the same setting, meaning a medical ward.

No other significant differences were observed according to BMI in simple indexes of thrombotic cascade activation (platelets and $\mathrm{D}$-dimer), metabolic decompensation (basal and maximal glucose levels), cardiac suffering and heart failure (troponin I and brain natriuretic peptide), and kidney (creatinine) or liver (aspartate aminotransferase, alanine aminotransferase, and gamma-glutamyl transferase) injury. Thus, we can assume that the severity of the disease in our patients with overweight and obesity was mostly related to the higher inflammatory activation.

One interesting aspect of our data is the absence of a direct clear relationship between the degree of adiposity, here imperfectly represented by the BMI, and the severity of COVID-19. Indeed, in our study, both the outcomes of the disease and the biochemical signs of inflammatory activation were not linearly related to BMI values, but they appeared to be worst in patients with overweight than with obesity. This could be in accordance with recent observations showing that the severity of COVID-19 was more related to increased visceral fat accumulation [10] or to the presence of metabolic syndrome [11] than to BMI strictly speaking. In summary, the occurrence of the "cytokine storm" and the consequent shift to a more severe COVID-19 could be more frequent in patients with adipose tissue-based chronic inflammation, visceral obesity, and metabolically disturbances than in patients with simply increased body fat. This could be in contrast to what has been observed in other settings of care, namely, in patients in the ICU [7], in which BMI directly correlates with a worse outcome.

One important limitation of our study is the lack of information regarding fat distribution in our patients with overweight and obesity. We were not able to collect waist circumference during the routine clinical activities in the COVID-19 ward, and other indexes of visceral adiposity were not available. Alternatively, the lack of difference between overweight and obesity in our population could be linked to the absence of a marked divergence in BMI values between the 2 groups. In fact, patients with obesity in our study were mostly classified into the first class of obesity (median BMI 31.7 [IQR 30.4-34.4] kg/ $\mathrm{m}^{2}$ ). The population of patients with more severe forms of obesity is therefore underrepresented in our sample.

In conclusion, our study confirms the link between overweight and obesity and the severity of COVID-19 and sup- ports the hypothesis that this association could be mediated by more sustained activation of inflammatory pathways. BMI may be not sufficient to recognize patients at higher risk of developing a more severe COVID-19. To achieve better risk prediction, further studies should focus more on phenotyping beyond BMI (i.e., body fat distribution).

\section{Statement of Ethics}

The research was conducted ethically in accordance with the World Medical Association Declaration of Helsinki. The study complied with standard operating procedures in place, in accordance with the European Data Protection Directive (95/46/EC) and, upon its entry into force, Regulation (EU) 2016/679 (also referred to as the General Data Protection Regulation [GDPR]). The study was a retrospective cohort study not involving any active treatment or procedure outside the normal clinical care and conducted by collecting anonymized patient data from electronic medical records. Informed consent for participation in the study was not required in accordance with national regulation on retrospective study. The study protocol was notified to the local institutional review board (Comitato Etico per la Sperimentazione Clinica della Provincia di Padova) (Prot. No. 0031090) on May 20, 2020 , and the need for patient's informed consent was waived.

\section{Conflict of Interest Statement}

The authors have no conflicts of interest to declare.

\section{Funding Sources}

The study was supported by the University of Padova Department of Medicine with a grant from Fondazione CA.RI.PA.RO (COVIDIMED project), Italy.

\section{Author Contributions}

S.B., R.V., and L.B. were involved in conceptualization; S.B. and L.B. were involved in formal analysis; G.B., C.S., C.D.P., R.F., and L.B. were involved in investigation and data collection; G.B. and C.S. were involved in data curation; S.B., G.B., and C.S. were involved in writing - original draft preparation; S.B., C.D.P., R.F., and L.B. were involved in writing - review and editing; R.V. and L.V. were involved in supervision. All authors have read and agreed to the published version of the manuscript.

\section{Data Availability Statement}

The original data source used for this study is not publicly available, since the data are part of the personal clinical documentation of the individual patients enrolled in the study, and they are protected by Italian privacy protection laws. An anonymized electronic database specific for this study is stored and locked in our center and available upon request. 


\section{References}

1 Popkin BM, Du S, Green WD, Beck MA, Algaith $\mathrm{T}$, Herbst $\mathrm{CH}$, et al. Individuals with obesity and COVID-19: a global perspective on the epidemiology and biological relationships. Obes Rev. 2020;21(11):e13128.

2 Docherty AB, Harrison EM, Green CA, Hardwick HE, Pius R, Norman L, et al. Features of 20133 UK patients in hospital with covid-19 using the ISARIC WHO clinical characterisation protocol: Prospective Observational Cohort Study. BMJ. 2020;369:m1985.

3 Deng M, Qi Y, Deng L, Wang H, Xu Y, Li Z, et al. Obesity as a potential predictor of disease severity in young COVID-19 patients: a Retrospective Study. Obesity. 2020;28(10): 1815-25.
4 Lighter J, Phillips M, Hochman S, Sterling S, Johnson D, Francois F, et al. Obesity in patients younger than 60 years is a risk factor for COVID-19 hospital admission. Clin Infect Dis. 2020;71(15):896-7.

5 Goossens GH, Dicker D, Farpour-Lambert NJ, Frühbeck G, Mullerova D, Woodward E, et al. Obesity and COVID-19: a perspective from the European Association for the Study of obesity on immunological perturbations, therapeutic challenges, and opportunities in obesity. Obes Facts. 2020;13(4):439-52.

6 Busetto L, Bettini S, Fabris R, Serra R, Dal Pra C, Maffei P, et al. Obesity and COVID-19: an Italian snapshot. Obesity 2020;28(9):1600-5.

7 Simonnet A, Chetboun M, Poissy J, Raverdy V, Noulette J, Duhamel A, et al. High prevalence of obesity in severe acute respiratory syndrome coronavirus-2 (SARS-CoV-2) requiring invasive mechanical ventilation. Obesity. 2020;28(7):1195-9.
8 Chen G, Wu D, Guo W, Cao Y, Huang D, Wang $\mathrm{H}$, et al. Clinical and immunological features of severe and moderate coronavirus disease 2019. J Clin Invest. 2020;130(5):26209.

9 Kanneganti TD, Dixit VD. Immunological complications of obesity. Nat Immunol. 2012; 13(8):707-12.

10 Watanabe M, Caruso D, Tuccinardi D, Risi R, Zerunian M, Polici M, et al. Visceral fat shows the strongest association with the need of intensive care in patients with COVID-19. Metabolism. 2020;111:154319.

11 Xie J, Zu Y, Alkhatib A, Pham TT, Gill F, Jang A, et al. Metabolic syndrome and COVID-19 mortality among adult black patients in New Orleans. Diabetes Care. 2020;44(1):188-93. 